\title{
Study on Microscopic Simulation Technology of Operation Process of Unmanned Vehicle Based on Virtual Track
}

\author{
Shiyao Teng ${ }^{1}$, Jinjin Tang ${ }^{1 *}$ \\ ${ }^{1}$ School of Traffic and Transportation, Beijing Jiaotong University, No. 3 Shangyuan Village, Haidian District, Beijing, 100044, \\ China
}

\begin{abstract}
With the continuous development of unmanned driving technology, more and more unmanned vehicles are on the road to start testing, under this circumstance, how to control these unmanned vehicles has become an urgent issue. Based on the basic idea of virtual track and cellular automata model, this paper researches and designs a microscopic simulation system of the operation process of unmanned vehicles. The aim of this paper is to use the idea of strong controllability of railways to control unmanned vehicles in the form of train diagram to ensure their orderly safety on the road. The stability and correctness of the system are verified through case analysis. At the end of the article, the future research direction is prospected.
\end{abstract}

\section{Introduction}

The popularization of cars makes our travel more convenient and faster, but the ensuing traffic congestion and traffic accidents also cause more loss of life and property. With the progress of science and technology and the development of artificial intelligence, unmanned vehicles $^{[1][2]}$ have appeared. Compared with human beings, unmanned vehicles are faster, more responsive, less affected by the environment, and they can avoid traffic accidents caused by human factors. In addition, unmanned vehicles can accurately identify traffic signals and road markings and strictly follow the preset procedures ${ }^{[3]}$. Thus unmanned vehicles make driving more standardized and traffic jams be avoided.

However, the operation of unmanned vehicles also faces some difficulties. Unmanned vehicles need to deal with the irregular driving behavior of other vehicles during the process of driving. In addition, how to obtain environmental information more accurately under the changeable and unpredictable traffic conditions ${ }^{[4]-[6]}$ also brings great challenges to unmanned vehicles.

In view of the above problems, virtual track is considered to be applied to the management of unmanned vehicles, and the idea of strong control of railway is used to carry out the control of unmanned vehicles in the way of operation diagram, so as to ensure the order and safety of unmanned vehicles more effectively. The actual road network on the ground is used as a virtual track to guide the unmanned vehicles. A number of virtual points are created with the safe distance as the interval, and the line length is the safe distance. According to OD requirements, the path is arranged for the unmanned vehicles, and then the vehicle operation is studied through microscopic simulation. Therefore, the microscopic simulation system of unmanned vehicle running process based on virtual track needs to be designed by software program.

\section{Design of Microscopic Simulation System for Operation Process of Unmanned Vehicle Based on Virtual Track}

\subsection{The system structure and function division}

This system uses the structural design diagram shown in Figure 1, including three parts: input module, simulation module and output module. 


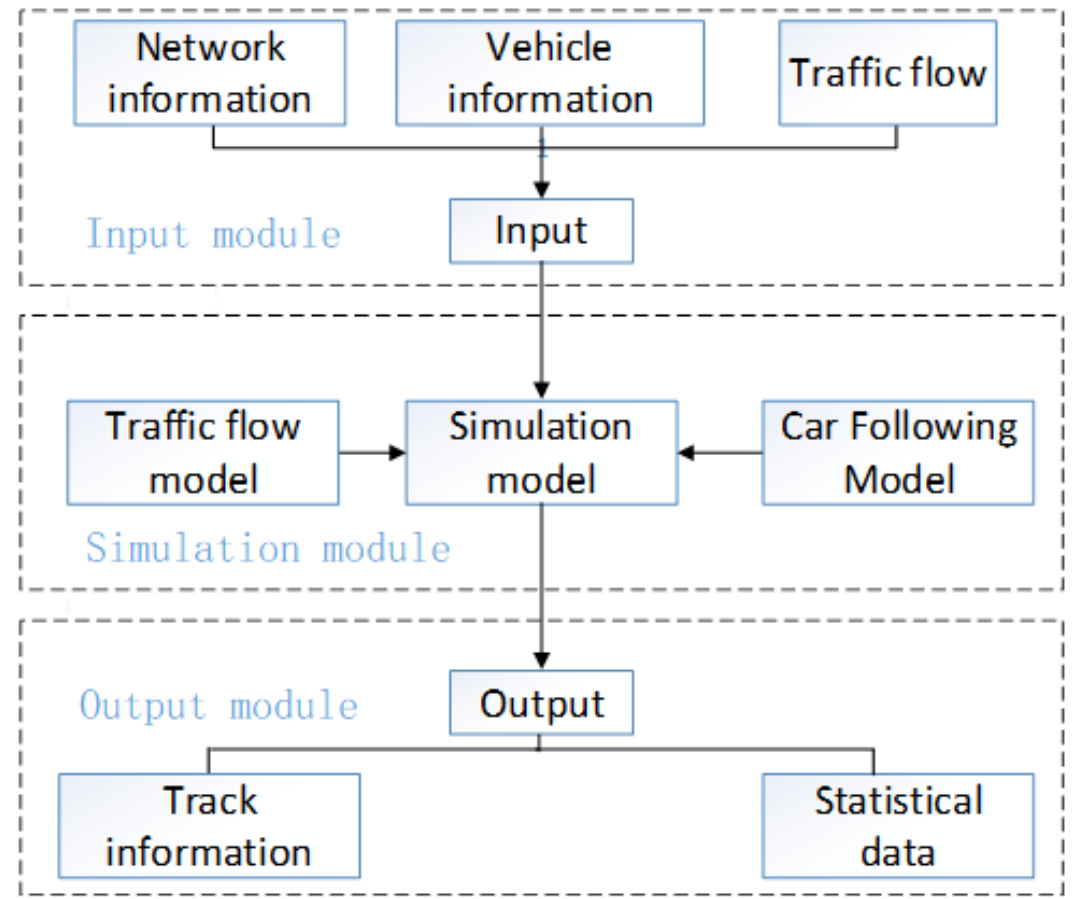

Figure 1. The diagram of KW(T) model.

It is not difficult to find out from the above Figure 1 that the microscopic simulation system of unmanned vehicle running process needs to have the following functions:

(1) The function of reading the road network in 'shp' format, reading the composition of the vehicle, and reading the mutual attraction between the communities;

(2) The function of generating OD demand between traffic zones through the gravity model;

(3) The function of using reasonable traffic distribution procedures to distribute the obtained OD traffic to the network, and generating the physical path of each agent based on the distribution result;
(4) The function of using a reasonable car following model to simulate the path of an individual vehicle;

(5) The function of outputting the trajectory information of individual vehicles and outputting the overall statistical data of vehicles;

\subsection{The design of road network format}

According to KW (T) model, the $\delta$ and $\tau$ between two adjacent vehicles are shown in Figure 2, where $\delta$ refers to the minimum space interval and $\tau$ refers to the minimum time interval.

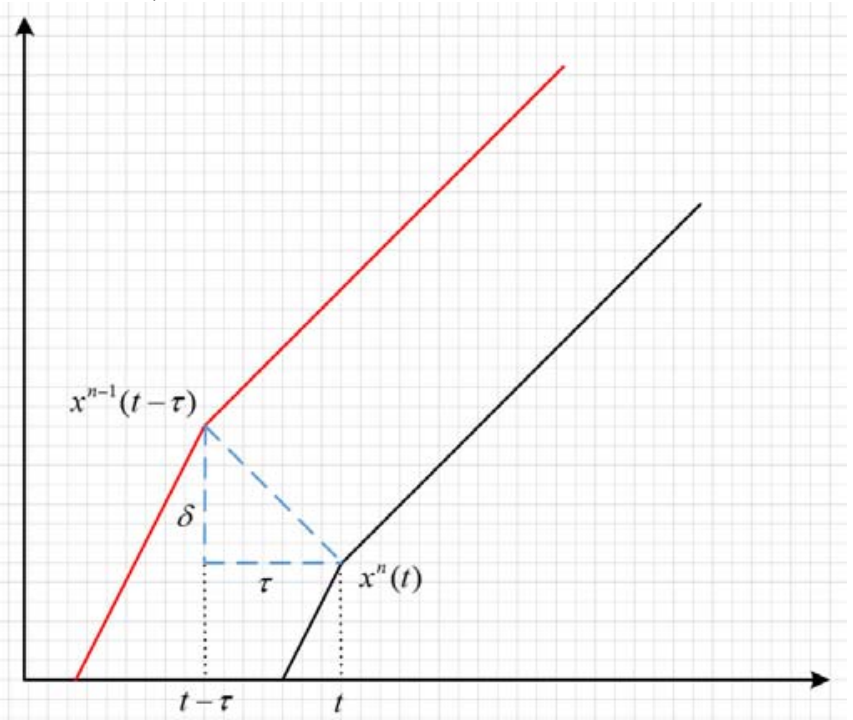

Figure 2. The system structure drawing.

Take $\delta$ as the length of a cell unit and cut the network according to the unit cell, where $\delta$ is $7 \mathrm{~m}$. The length of each link in the original road network is calculated according to the coordinate information of the starting point and the ending point. The number of segments of a link is determined according to the total length of each link. Each link can be decomposed into the number of cells on average and all original links are 
decomposed. Since the length of each link calculated may not be an integer, the length of each cell is a noninteger approximate to $7 \mathrm{~m}$.

\subsection{Applied technology and programming environment}

Based on the requirement of future application in largescale road network test, the language used in the design process of the system is $\mathrm{C}++$ language.

System development, operation and other information are as follows:

(1) Development environment: Visual_Studio2015;

(2) Operating environment: Windows_10 Home Chinese version;

(3) RAM: 8.00GB

(4) Processor: Intel (R) _Core (TM) i5-4200H CPU@2.80GHz

\subsection{Algorithm design}

According to the division of system mechanism and function, the algorithm flow of microscopic simulation program is designed as shown in Figure 3.

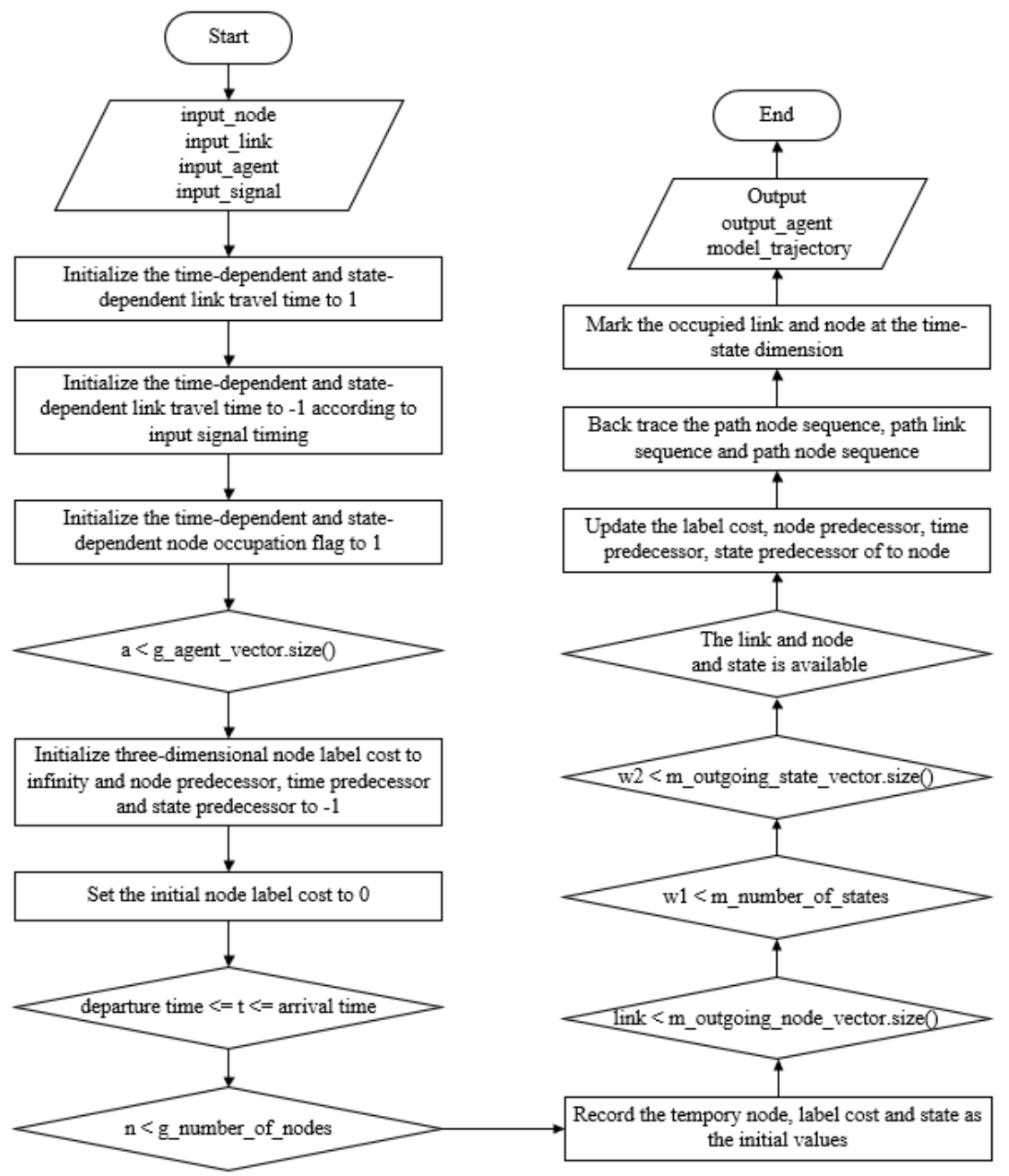

Figure 3 Algorithm flow. the other agents. to 0 . and put the starting point in the SElist. from the SElist. of to_node and to_link. SElist. of the ending point.

Step1: Initialize label cost first, set the label cost of all time and space state points to infinity, and set the predecessor of node, time, and state to -1 . This item is generally done by $\mathrm{dp}$, in order to avoid the influence of

Step2: Set the label_cost of the selected starting point

Step3: For each t: Clear the SElist (scan eligible list),

Step4: For each node: Get the starting point number

Step5: For each $\mathrm{m}$ _outgoing node: Get the number

Step6: First judge whether cost is sufficient to meet the requirements; Second, judge whether arrival time and travel time meet the requirements; Finally, if temporary_label_cost is less than m label_cost and the time also meets the requirements, update the label_cost of this point and record the predecessor of node, time and state. And go back to Step 5 to put the to_node into

Step7: Trace the shortest path according to label_cost 


\section{Case analysis}

\subsection{The Basic test network}

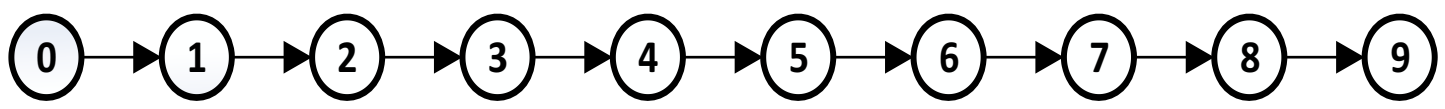

Figure 4. The basic test network structure.

Enter the agent data as shown in Table 1. Both agents (Agent1 and Agent2) are at time 0 (note: the starting point of time here is 0 ) from the starting point 0 to the ending point 9 . Node 0 and node 9 are two zones numbered 2001 and 2002 respectively. Set the traffic volume of zone node 2001 to 2 , traffic attraction volume

Table 1. The departure and arrival information.

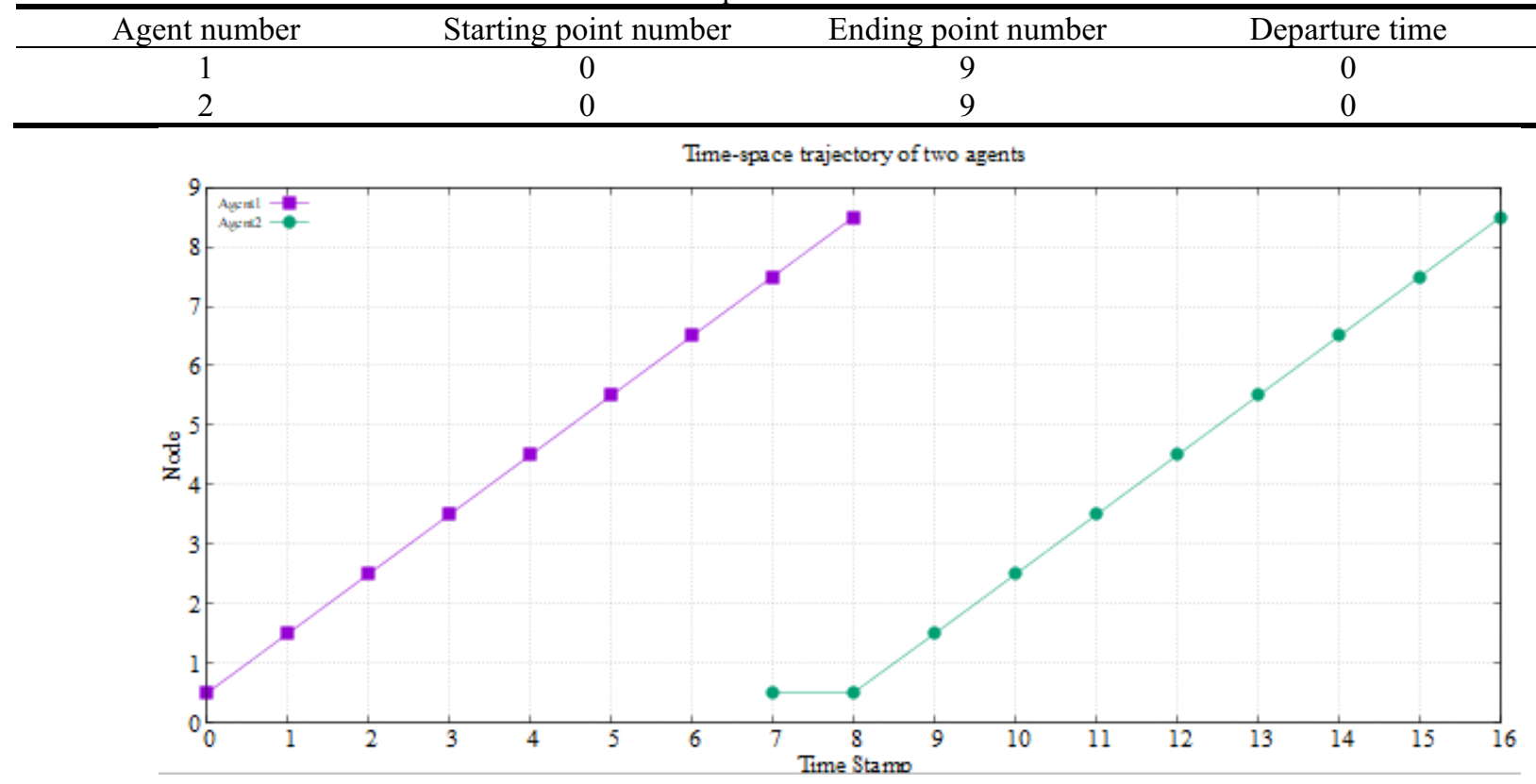

Figure 5. The time-space trajectory of the two agents.

\subsection{The Basic test network with merging}

Assume that the merging behavior is introduced in the behavior

Figure 6 below.

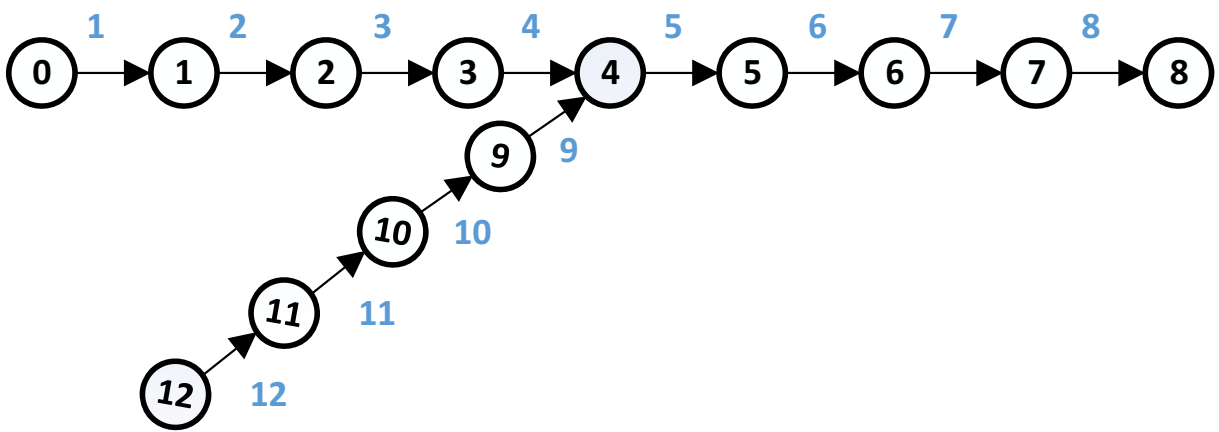

Figure 6. The basic test network with merging behavior structure.

Enter the agent data as shown in Table 2. Both agents (Agent1 and Agent2) are at time 0 (note: the starting point of time here is 0 ) from the starting point 0 and starting point 12 to the ending point 8 . Node 0 , node 12 , and node 8 are three zones numbered 2001, 2002 and 2003 respectively. Set the traffic volume of the zone node 2001 to 1 , the traffic attraction volume of the zone node 2001 to 0 , the traffic volume of the zone node 2002 to 1 , the traffic attraction volume of the zone node 2002 to 0 , the traffic volume of the zone node 2003 to 0 , and the traffic attraction volume of the zone node 2003 to 2 . The time-space trajectory of the two agents obtained by program simulation is shown in Figure 7. 
Table 2. The departure and arrival information.

\begin{tabular}{ccccc}
\hline Agent number & Starting point number & Ending point number & Departure time \\
\hline 1 & 0 & 8 & 0 \\
\hline
\end{tabular}

Figure 7. The time-space trajectory of the two agents.

\subsection{Actual road network}

Malian Road is located in the west of Xuanwu District, Beijing, with West Third Ring Road in the west,
Guanganmenwai Street in the north, across the street from Beijing West Railway Station, and Lize Road in the south. The road network after opening and cutting in NEXTA is shown in Figure 8.

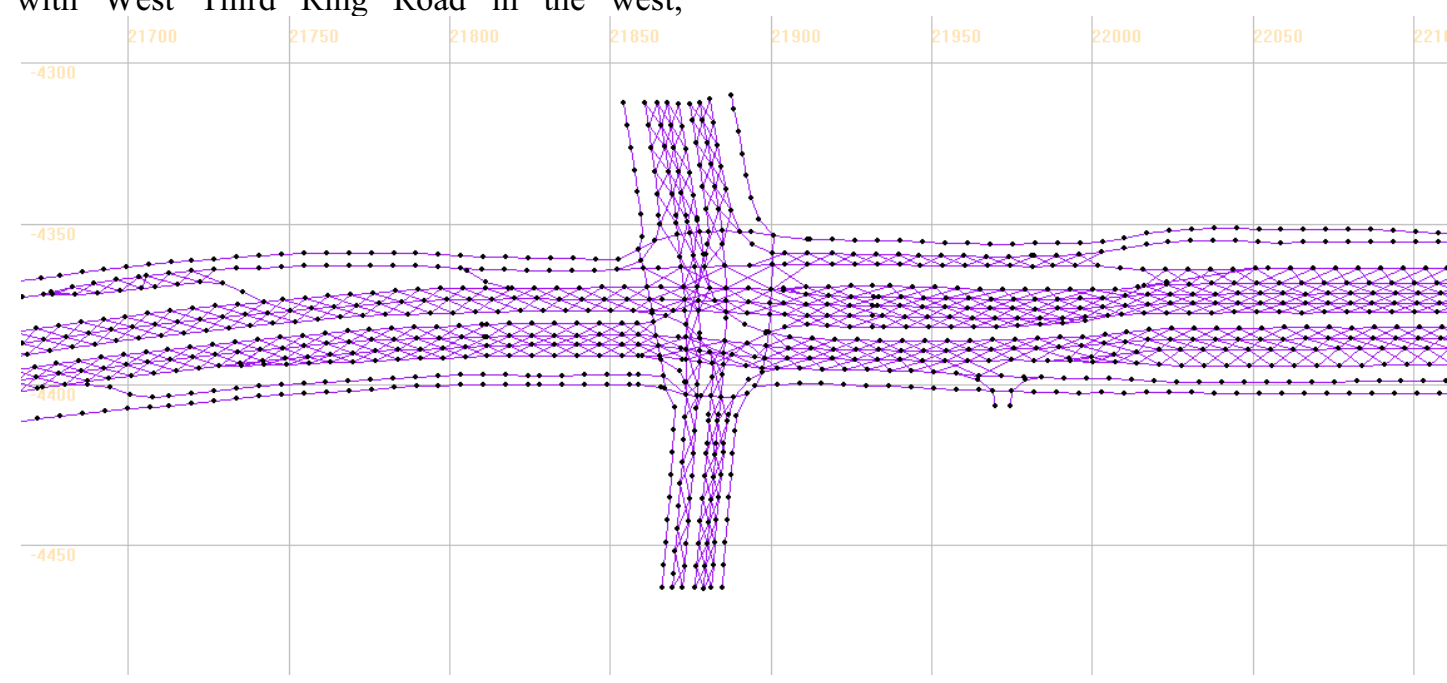

Figure 8. Malian road network in NEXTA program

The program shows that there are 1505 nodes and 2579 arcs in the Malian road network by reading the Malian road network files. A total of 28 zones are set in input_activity_location.csv. The number of vehicles generated by the program according to the gravity model is 3312, and the CPU takes an average of 25 seconds through multiple tests. The output_agent.csv file is shown in Figure 9 below. The complete value is $c$, which can indicate that the unmanned vehicles have reached their destinations. 


\begin{tabular}{|c|c|c|c|c|c|c|c|c|c|c|c|c|c|}
\hline agent_id & tour & lepe & dur & fol: & from_zone & zone_i & m_oris & destir & arture & arture & ival_t & ival_t & complete_flag \\
\hline 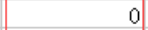 & -1 & -1 & -1 & -1 & 2001 & 2008 & 1178 & 1296 & 0 & 0 & 0.25 & 15.2 & $=$ \\
\hline 1 & -1 & -1 & -1 & -1 & 2001 & 2008 & 1178 & 1296 & 0.01 & 0.8 & 0.34 & 20.2 & $c$ \\
\hline 2 & -1 & -1 & -1 & -1 & 2001 & 2008 & 1178 & 1296 & 0.03 & 1.6 & 0.59 & 35.6 & $c$ \\
\hline 3 & -1 & -1 & -1 & -1 & 2001 & 2008 & 1178 & 1296 & 0.04 & 2.4 & 0.68 & 41 & c \\
\hline 4 & -1 & -1 & -1 & -1 & 2001 & 2008 & 1178 & 1296 & 0.05 & 3.2 & 0.71 & 42.6 & c \\
\hline 5 & -1 & -1 & -1 & -1 & 2001 & 2008 & 1178 & 1296 & 0.07 & 4 & 0.74 & 44.2 & $c$ \\
\hline 6 & -1 & -1 & -1 & -1 & 2001 & 2008 & 1178 & 1296 & 0.08 & 4.8 & 0.79 & 47.6 & $c$ \\
\hline 7 & -1 & -1 & -1 & -1 & 2001 & 2008 & 1178 & 1296 & 0.09 & 5.6 & 0.82 & 49.2 & $c$ \\
\hline 8 & -1 & -1 & -1 & -1 & 2001 & 2008 & 1178 & 1296 & 0.11 & 6.4 & 0.85 & 50.8 & $c$ \\
\hline 9 & -1 & -1 & -1 & -1 & 2001 & 2008 & 1178 & 1296 & 0.12 & 7.2 & 0.9 & 54.2 & $c$ \\
\hline 10 & -1 & -1 & -1 & -1 & 2001 & 2008 & 1178 & 1296 & 0.13 & 8 & 0.93 & 55.8 & $c$ \\
\hline 11 & -1 & -1 & -1 & -1 & 2001 & 2008 & 1178 & 1296 & 0.15 & 8.8 & 0.96 & 57.4 & $=$ \\
\hline 12 & -1 & -1 & -1 & -1 & 2001 & 2008 & 1178 & 1296 & 0.16 & 9.6 & 0.98 & 59 & $c$ \\
\hline 13 & -1 & -1 & -1 & -1 & 2001 & 2008 & 1178 & 1296 & 0.17 & 10.4 & 1.01 & 60.6 & $=$ \\
\hline 14 & -1 & -1 & -1 & -1 & 2001 & 2008 & 1178 & 1296 & 0.19 & 11.2 & 1.04 & 62.2 & $c$ \\
\hline 15 & -1 & -1 & -1 & -1 & 2001 & 2008 & 1178 & 1296 & 0.2 & 12 & 1.06 & 63.8 & $c$ \\
\hline 16 & -1 & -1 & -1 & -1 & 2001 & 2008 & 1178 & 1296 & 0.21 & 12.8 & 1.09 & 65.4 & $=$ \\
\hline 17 & -1 & -1 & -1 & -1 & 2001 & 2008 & 1178 & 1296 & 0.23 & 13.6 & 1.12 & 67 & $c$ \\
\hline 18 & -1 & -1 & -1 & -1 & 2001 & 2008 & 1178 & 1296 & 0.24 & 14. 4 & 1.14 & 68.6 & $c$ \\
\hline 19 & -1 & -1 & -1 & -1 & 2001 & 2008 & 1178 & 1296 & 0.25 & 15.2 & 1.17 & 70.2 & $c$ \\
\hline 20 & -1 & -1 & -1 & -1 & 2001 & 2008 & 1178 & 1296 & 0.27 & 16 & 1.2 & 71.8 & $c$ \\
\hline 21 & -1 & -1 & -1 & -1 & 2001 & 2008 & 1178 & 1296 & 0.28 & 16.8 & 1.22 & 73.4 & $c$ \\
\hline 22 & -1 & -1 & -1 & -1 & 2001 & 2008 & 1178 & 1296 & 0.29 & 17.6 & 1.28 & 76.6 & 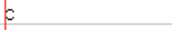 \\
\hline 23 & -1 & -1 & -1 & -1 & 2001 & 2008 & 1178 & 1296 & 0.31 & 18.4 & 1.33 & 79.8 & c \\
\hline 24 & -1 & -1 & -1 & -1 & 2001 & 2009 & 1178 & 1482 & 0.32 & 19.2 & 0.67 & 40.2 & $=$ \\
\hline 25 & -1 & -1 & -1 & -1 & 2001 & 2009 & 1178 & 1482 & 0.33 & 20 & 0.7 & 41.8 & $c$ \\
\hline
\end{tabular}

Figure 9. The output agent.csv file

\section{Conclusion}

In the context of the rapid development of unmanned vehicles, this paper combines the basic ideas of virtual track and railway control to conduct microscopic simulation research on the operation process of unmanned vehicles. At the same time, related functions are realized through the simulation system, and then the planning and control suggestions for unmanned vehicles are proposed.

It will be a long process for unmanned cars to replace drivers. The popularization of unmanned cars will make cities more intelligent. In the future, there is an opportunity to have a centralized scheduling system to optimize the population of the same destination and promote Shared travel.

\section{References}

1. Li, Y., Ni, J., Hu, J., Pan, B. (2018) The Design of Driverless Vehicle Trajectory Tracking Control Strategy. IFAC-PapersOnLine, 51(31), 738-745.

2. Campbell, S., Naeem, W., Irwin, G.W. (2012) A review on improving the autonomy of unmanned surface vehicles through intelligent collision avoidance manoeuvres. Annual Reviews in Control, 36(2), 267-283.

3. Ding, D., Ding, Z., Wei, G., Han, F. (2019) An improved reinforcement learning algorithm based on knowledge transfer and applications in autonomous vehicles. Neurocomputing, 361, 243255.

4. Salonen, A.O. (2018) Passenger's subjective traffic safety, in-vehicle security and emergency management in the driverless shuttle bus in Finland. Transport Policy, 61, 106-110.

5. Rosell, J., Allen, J. (2020) Test-riding the driverless bus: Determinants of satisfaction and reuse intention in eight test-track locations. Transportation Research Part A: Policy and Practice, 140, 166-189.

6. Smolnicki, P.M., Sołtys, J. (2016) Driverless Mobility: The Impact on Metropolitan Spatial Structures. Procedia Engineering, 161, 2184-2190. 